\title{
Researchers oppose data-sharing proposal
}

S everal hundred clinical investigators around the world oppose a proposal by medical journal editors to make clinical trial data freely available to other researchers.

Two research consortia representing the triallists recently made their cases in the New England Journal of Medicine. They were responding to the proposal published in January by the International Committee of Medical Journal Editors (ICMJE) that, as a condition of publication and as part of a clinical trial's registration, researchers agree to share the anonymized individual patient data underlying their reports within six months of publication. The proposal was simultaneously published in 14 medical journals, including CMAJ.

Neither triallist group opposed the idea of data-sharing in principle, but both said the proposed six-month interval from publication to data availability is too short.

The International Consortium of Investigators for Fairness in Trial Data Sharing, based at McMaster University and representing 282 investigators in 33 countries, contends that the ICMJE proposal could disadvantage researchers who participate in trials.

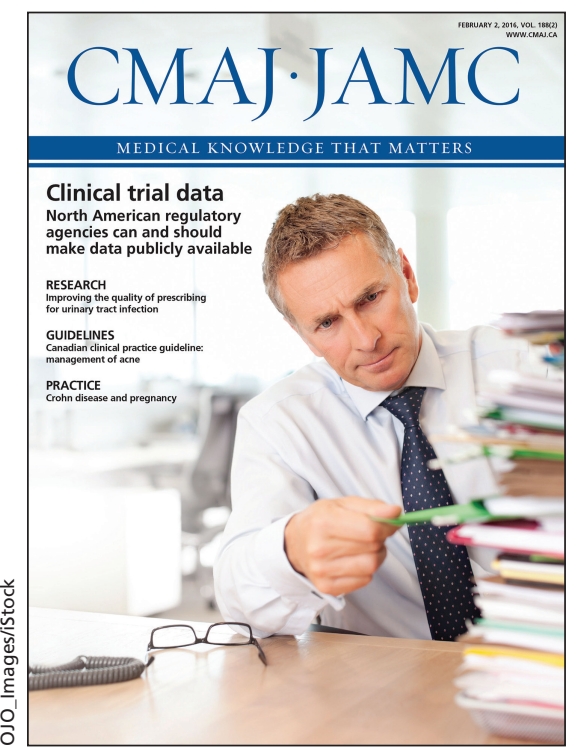

A proposal by medical journal editors to make clinical research freely available was published in $\mathbf{1 4}$ medical journals, including CMAJ.

study have the first opportunity to publish on the data that was generated from the studies that we conducted and based upon the money that we helped to invest to get those trials done?"

Devereaux, a professor of clinical epidemiology and biostatistics, suggested that some non-participant secondary analyses could be done by opportunists.

\section{The proposed six-month interval from publication to data availability is too short.}

\footnotetext{
"Most of our grants are funded by peer-reviewers like CIHR [Canadian Institutes of Health Research], but we have to cross-subsidize those grants to a million-plus dollars to get those trials done," said Dr. PJ Devereaux of the McMaster group.

"Is it really fair that we should be the ones footing all those bills and then simply handing the data over to someone else? Or is it not appropriate that the people who were involved in the
}

"From an academic productivity point of view, why bother doing any of the heavy lifting? Just grab everything six months after the fact, and start publishing on it."

To allow the original investigators time to do and publish all the secondary analyses they intended, they should be allowed exclusive use of the data for at least two years after publication of the primary trial results and an additional six months for every year it took to complete the trial, to a maximum of five years, according to the group.

The second group, the Academic Research Organization Consortium for Continuing Evaluation of Scientific Studies - Cardiovascular (or ACCESS $\mathrm{CV}$ ), offered its own proposal for sharing data from cardiovascular trials one for which issues of resources, funding and assigning "meaningful academic credit" to those who designed and conducted the original research have not yet been addressed.

The authors - led by Dr. Manesh Patel, associate professor of medicine at Duke University, and representing 265 signatories from 44 countries suggested that data requests be considered by a group of ACCESS CV members not involved in the trial, along with the principal investigator, a trial statistician and a member of the dataand-safety monitoring board. The interval between first publication and access should be 24 months to allow time to build "secure access to the database" and to allow investigators to complete secondary analyses.

The points made by the two research consortia, however, were already included in the feedback received in response to the original proposal, said Dr. Darren Taichman, executive deputy editor of the Annals of Internal Medicine and corresponding author on the original data-sharing proposal.

"The ICMJE has sought broad input from the many stakeholders involved in data sharing, including researchers who conduct clinical trials, sponsors, trial participants and others," he said in an email. "The ICMJE is carefully studying these issues as it pursues policies that will achieve everyone's goal: improving care by maximizing the knowledge gained from clinical trial participants' generous efforts." - Terry Murray, Toronto, Ont.

CMAJ 2016. DOI:10.1503/cmaj.109-5317 